\title{
Exposure to general anesthesia and the risk of dementia
}

\author{
Chih-Wen Yang ${ }^{1,2}$ \\ Jong-Ling Fuh',3 \\ 'Department of Neurology, National \\ Yang-Ming University Hospital, Ilan, \\ ${ }^{2}$ National Yang-Ming University \\ School of Medicine, ${ }^{3}$ Department of \\ Neurology, Neurological Institute, \\ Taipei Veterans General Hospital, \\ Taipei, Taiwan, Republic of China
}

\author{
This article was published in the following Dove Press journal: \\ Journal of Pain Research \\ 16 October 2015 \\ Number of times this article has been viewed
}

Correspondence: Jong-Ling Fuh Department of Neurology, Neurological Institute, Taipei Veterans General Hospital, No 201 Section 2, Shipai Road, Taipei I I2, Taiwan, Republic of China Tel +8862 28752522

Fax +8862287652I5

Email jlfuh@vghtpe.gov.tw
Abstract: Exposure to anesthesia and surgery has been hypothesized to increase the risk of developing Alzheimer's disease (AD). While the exact pathogenesis of $\mathrm{AD}$ remains unknown, it potentially involves specific proteins (eg, amyloid beta and tau) and neuroinflammation. A growing body of preclinical evidence also suggests that anesthetic agents interact with the components that mediate AD neuropathology at multiple levels. However, it remains unclear whether anesthesia and surgery are associated with an increased risk of AD in humans. To date, there have not been randomized controlled trials to provide evidence for such a causal relationship. Besides, observational studies showed inconsistent results. A meta-analysis of 15 case-control studies revealed no statistically significant association between general anesthesia and the development of $\mathrm{AD}$ (pooled odds ratio $[\mathrm{OR}]=1.05 ; P=0.43$ ). However, a few retrospective cohort studies have demonstrated that exposure to anesthesia and surgery is associated with an increased risk of AD. Thus, well-designed studies with longer follow-up periods are still needed to define the role of anesthesia in relation to the development of AD.

Keywords: anesthesia, surgery, dementia, Alzheimer's disease

\section{Introduction}

Dementia is becoming increasingly prevalent among the elderly population. In 2001, $\sim 24$ million people worldwide were afflicted with dementia, and that number is estimated to double to an estimated prevalence of 42 million by 2020 and 81 million by $2040 .{ }^{1}$ Dementia is challenging from a medical care perspective since it is both progressive and irreversible. Additionally, dementia is a leading cause of admission to long-term care facilities and a major risk factor for hospitalization. ${ }^{2}$ Alzheimer's disease (AD) is the most common form of dementia, and it constitutes approximately two-thirds of all cases. ${ }^{3}$ It is currently hypothesized that the pathophysiology of AD involves the accumulation of amyloid beta $(A \beta)$ proteins and the hyperphosphorylation of tau proteins, which leads to neurofibrillary tangles and neuronal loss in the brain. ${ }^{4}$ However, AD pathogenesis is multifactorial, whereby both genetic susceptibility and environmental factors contribute to neurodegeneration.

Generally considered safe and effective, anesthetic drugs have bestowed enormous clinical benefits. Besides, with the global acceleration of population aging, surgery is being performed more frequently. Correspondingly, there is growing concern that anesthesia and/or surgery may have neurodegenerative complications. In vitro studies have shown that inhaled anesthetic agents can promote $A \beta$ oligomerization and enhance $A \beta$-induced neurotoxicity. ${ }^{5,6}$ Animal studies have also provided evidence that exposure to anesthetic drugs can impair memory, ${ }^{7}$ can induce caspase- 3 activation, and 
can increase levels of $A \beta{ }^{8,9}$ Postoperative cognitive decline (POCD) in humans is generally considered to be a shortlived condition where normal function returns within days, while cognitive changes may persist for weeks or longer. ${ }^{10,11}$ It remains unclear whether this decline simply unmasks the early stages of dementia or if it is a predictor of later dementia. Regardless, with clinical features similar to those observed in patients with dementia, the incidence of longterm postoperative cognitive impairment raises concerns that anesthesia and surgery may accelerate the onset and progression of dementia. ${ }^{12}$ Currently, it remains controversial as to whether anesthesia is associated with an elevated risk of developing AD in human populations. Observational studies have reported inconsistent results to support the causal relationship between anesthesia, surgery, and dementia. ${ }^{13-16}$ Accordingly, the consensus statement from the First International Workshop on Anesthetics and Alzheimer's Disease in 2009 suggested that statistically sound prospective and retrospective human studies of the risk of AD after anesthesia are needed. ${ }^{17}$ Therefore, the purpose of this review is to summarize the preclinical and clinical evidence regarding the capacity for anesthesia and surgery to induce or accelerate neurodegeneration in $\mathrm{AD}$ in order to provide perspective regarding additional studies that are needed.

\section{Preclinical studies Effects of anesthetics on $A \beta$ levels}

$\mathrm{AD}$ is pathologically characterized by extracellular amyloid plaques and intraneuronal neurofibrillary tangles, both of which comprise filaments that are densely packed and highly insoluble. However, there are soluble elements in these abnormal structures, and they include $A \beta$ peptides and tau proteins, respectively. ${ }^{18} \mathrm{~A} \beta$ peptides consist of 36-43 amino acids, and they are natural products of metabolism. A $\beta$ peptides derive from the proteolysis of amyloid precursor protein (APPs) by $\beta$-site APP-cleaving enzymes (BACEs) and $\gamma$-secretases. ${ }^{19}$ The resulting $A \beta$ peptides self-assemble into multiple physical forms. Soluble A $\beta$ oligomers can coalesce into intermediate assemblies, and both states are neurotoxic forms. It has been shown that $\mathrm{A} \beta 42$ oligomers induce oxidative damage, promote tau hyperphosphorylation, and then result in toxic effects on synapses. ${ }^{20} \mathrm{~A} \beta$ can also aggregate into fibrils which arrange themselves into $\beta$ sheets to form the insoluble fibers of amyloid plaques. ${ }^{21}$ To date, the "amyloid hypothesis" is the most common theory regarding $\mathrm{AD}$ pathogenesis, and it is based on studies of the genetic forms of $\mathrm{AD}$ and evidence of the cellular toxicity of $A \beta$. This hypothesis states that an imbalance between $A \beta$ protein production and clearance leads to an excessive accumulation of $A \beta$ protein, and subsequently, neurodegeneration is observed. ${ }^{22}$ The inhaled anesthetics, isoflurane $(1.2 \%$ and $2.5 \%)$ and halothane $(0.8 \%$ and $1.5 \%$ ), have been found to enhance $A \beta$ oligomerization and to potentiate $A \beta$-induced cytotoxicity in rat pheochromocytoma cells. ${ }^{5,23}$ In vitro multidimensional nuclear magnetic resonance spectroscopy has also demonstrated that isoflurane, halothane, and propofol interact with $A \beta$ and induce $A \beta$ oligomerization. ${ }^{24,25}$ In human neuroglioma cells that were stably transfected with full-length APP, isoflurane ( $2 \%$ for 6 hours) and sevoflurane ( $4.1 \%$ for 6 hours) induced caspase activation, cell death, and an accumulation of extracellular $A \beta{ }^{26,27}$ Thus, it has been proposed that inhaled anesthetics induce caspase activation and apoptosis by disrupting intracellular calcium homeostasis. ${ }^{28}$ Moreover, the data from in vitro studies suggested a potential pathway of inhaled anesthetics-related neurotoxicity; that inhaled anesthetics induced caspase activation and apoptosis, which led to increased levels of BACE and $\gamma$-secretase and the generation of $A \beta$ from $A P P .{ }^{27,29}$ The generated $A \beta$ may further potentiate the isoflurane-induced caspase activation and apoptosis..$^{30}$ Although these studies proposed that administration of isoflurane has been shown to induce $A \beta$ accumulation and neurotoxicity via an apoptosis-related pathway, there is currently no satisfactory way to confirm these mechanisms in aging human brains.

In animal studies of 12-month-old transgenic mice with $\mathrm{AD}$, administration of halothane $(0.8 \%-1 \%)$ resulted in greater amyloidopathy compared with either isoflurane $(0.9 \%-1 \%)$ or control condition. ${ }^{31}$ The administration of isoflurane ( $1.4 \%$ for 2 hours) and sevoflurane ( $2.5 \%$ for 2 hours) have also been shown to induce caspase activation and apoptosis and to increase $\mathrm{A} \beta$ accumulation in 5-month-old wildtype mice. ${ }^{9,27}$ In another study that compared wild-type and transgenic AD mice, the latter are more susceptible to developing increased $\mathrm{A} \beta$ aggregation, apoptosis, and microglia activation following isoflurane anesthesia. ${ }^{32}$ Additionally, isoflurane exposure has been found to impair memory and learning in wild-type mice. ${ }^{31}$ Taken together, these results suggest that inhaled anesthetics may cause cognitive impairment and amyloidogenesis in vivo.

In contrast with isoflurane and sevoflurane, desflurane ( $12 \%$ for 6 hours) and nitrous oxide ( $70 \%$ for 6 hours) do not appear to cause apoptosis or $\mathrm{A} \beta$ accumulation in cultured cells or mouse primary neurons. ${ }^{33-35}$ Moreover, administration of desflurane has not been found to impair learning and memory in mice. ${ }^{36}$ However, additional studies are necessary 
to determine whether desflurane is superior to isoflurane or sevoflurane in regard to anesthetic-related neurotoxicity.

Collectively, preclinical studies suggest that certain anesthetic agents (eg, isoflurane and sevoflurane) may increase $\mathrm{A} \beta$ production and accumulation, and this phenomenon may be mediated via caspase activation and apoptosis. ${ }^{37}$ Further studies are warranted to determine the in vivo relevance of these in vitro findings.

\section{Effects of anesthetics on tau pathology}

Intraneuronal neurofibrillary tangles, which are composed of aberrantly hyperphosphorylated tau proteins, are one of the major neuropathological hallmarks of AD. Tau protein is normally enriched in the axonal compartment. However, during $\mathrm{AD}$, tau proteins become hyperphosphorylated, and they assemble into paired helical filaments that aggregate in the somatodendritic compartment of affected neurons. ${ }^{38}$ Accumulating evidence suggests that $A \beta$ and tau work together, independent of their accumulation into plaques and tangles, respectively, to induce neuronal dysfunction and damage. ${ }^{19}$ Moreover, it has been determined that $A \beta$ is dependent on the presence of tau for its neurotoxic properties. It has been suggested that $A \beta$ is upstream of tau in $A D$ pathogenesis and $A \beta$ induces the transformation of normal tau into a toxic form that subsequently participates in a feedback loop to enhance $\mathrm{A} \beta$ toxicity. ${ }^{39}$ Prolonged hypothermia induced by both intravenous (chloral hydrate $[500 \mathrm{mg} / \mathrm{kg}$ ] and sodium pentobarbital $[100 \mathrm{mg} / \mathrm{kg}])$ and inhaled anesthetics (isoflurane) was found to induce pronounced tau hyperphosphorylation in wild-type mice. ${ }^{8}$ However, this effect was reversed when the body temperature of the mice returned to normal. Moreover, there was no increase in tau phosphorylation when mice with a normal body temperature were exposed to isoflurane. ${ }^{40}$ Thus, anesthesia-induced hypothermia may be a mediator of tau hyperphosphorylation. Correspondingly, in a transgenic mouse model of tauopathy, the capacity of isoflurane $(1.3 \%)$ to increased accumulation of neurofibrillary tangles was found to be dependent on anesthesia-induced hypothermia rather than on exposure to isoflurane per se. ${ }^{41,42}$ Moreover, the impairment of memory following isoflurane-induced hypothermia has been associated with increases in tau phosphorylation in rodents. ${ }^{40}$ In addition to evidence suggesting that anesthesia-induced hypothermia can produce tau hyperphosphorylation and exacerbate neurofibrillary degeneration, in vitro and animal studies suggest that anesthesia under normothermic conditions can also lead to tau hyperphosphorylation. An increase in tau phosphorylation was observed in mice following administration of inhaled ether or intraperitoneal pentobarbital in normothermic condition. ${ }^{43}$ Exposure of propofol in normothermic cultured cells resulted in increased levels of phosphorylated tau. ${ }^{44}$ Tau hyperphosphorylation in rodent hippocampus occurred following normothermic intraperitoneal propofol or inhaled sevoflurane (1.5\% and $2.5 \%)$ administration. ${ }^{45,46}$ Interestingly, Le Freche et al also demonstrated that in mice that underwent repeated normothermic exposure to sevoflurane (1.5\% and $2.5 \%)$, spatial memory deficits and persistent tau hyperphosphorylation were observed, with the latter mediated via the activation of specific kinases. ${ }^{46}$ Furthermore, in transgenic AD mouse models, Tang et al demonstrated that repeated normothermic administration of halothane $(0.9 \%-1.1 \%)$ or isoflurane $(0.9 \%-1.1 \%)$ was associated with persistent hippocampal tau hyperphosphorylation. ${ }^{47}$

In summary, the results of the preclinical studies published to date suggest that certain anesthetics can exacerbate tau pathology. Specifically, anesthesia-induced hypothermia has led to tau hyperphosphorylation and the development of neurofibrillary pathology in transgenic mouse models. However, mechanisms other than hypothermia likely play a role in anesthesia-related tau pathology since changes in tau pathology have been described during the administration of anesthesia under normothermic conditions.

\section{Effects of anesthetics on neuroinflammation}

Accumulating evidence also supports the involvement of neuroinflammation in the smoldering pathogenesis of AD. Neuroinflammation is not merely a passive process that is activated by the accumulation of senile plaques and neurofibrillary tangles, but rather is a process that contributes to the pathogenesis of $\mathrm{AD}$ as much as the plaques and tangles that characterize AD. ${ }^{48}$ For example, neuroinflammation has been found to induce tau hyperphosphorylation and to promote the formation of neurofibrillary tangles in $\mathrm{AD}$ transgenic mice. ${ }^{49}$ It is also hypothesized that pathological protein aggregates of hyperphosphorylated tau, $A \beta$, small oligomers, or senile plaques activate astrocytes and microglia, the immune cells in the brain. ${ }^{50}$ Peripheral inflammatory cells can then be recruited to the central nervous system through either a compromised blood-brain barrier (BBB) or circumventricular organs. In addition, microglia may be "primed" for exaggerated responses to inflammatory signals that are induced by chronic and smoldering neurodegeneration that is associated with $\mathrm{AD}{ }^{51}$ The occurrence of an acute peripheral inflammatory event, such as surgery or infection, 
may further elicit a cytokine response in the brain that causes tau phosphorylation and microglial activation. ${ }^{52} \mathrm{~A}$ few potential mechanisms have been proposed regarding alterations in the inflammatory processes that occur due to anesthetics. For example, in a recent study, it was demonstrated that sevoflurane induces structural changes in brain vascular endothelial cells and increases BBB permeability in aged rats. ${ }^{53}$ Compromise of the $\mathrm{BBB}$ may enhance the infiltration of peripheral inflammatory cells to the brain. Administration of nitrous oxide has also been shown to alter monocyte recruitment in animals, ${ }^{54}$ while isoflurane and sevoflurane have been shown to directly interact with the signaling molecules in the inflammatory cascade, such as the integrins..$^{55,56}$ Emerging studies have further suggested that anesthetics may increase the levels of proinflammatory cytokines, which may cause neuroinflammation, leading to promotion of AD neuropathogenesis. ${ }^{57,58} \mathrm{Wu}$ et al detected an increase in the production of the proinflammatory cytokines, TNF- $\alpha$, IL-6, and IL-1 $\beta$, in the mouse brain following the administration of isoflurane anesthesia (1.2\% and 2.5\%). ${ }^{23}$ Importantly, isoflurane-induced production of neuroinflammatory cytokines primarily derives from neurons. Correspondingly, exposure to isoflurane $(1.2 \%$ or $1.4 \%)$ has been found to impair learning ability in aged rats, and a potential role for IL- $1 \beta$ in mediating this effect has been proposed. ${ }^{59}$ Furthermore, some anti-inflammation treatments that mitigate isofluraneinduced neuroinflammation have also been shown to improve long-lasting cognitive disorder. ${ }^{60}$

In summary, laboratory evidence has demonstrated that inhaled anesthetics alter BBB permeability and increase the recruitment of peripheral leukocytes to the central nervous system. In particular, isoflurane anesthesia has been found to promote proinflammatory cytokine production, transient neuroinflammation, and long-lasting cognitive disorder in aged rodent brains. However, it remains unclear whether isoflurane-induced neuroinflammation can trigger tau hyperphosphorylation to drive AD pathology.

\section{Effects of surgery on cognition}

In clinical practice, it is often difficult to separate the impact of anesthesia from that of surgery on cognitive impairment in humans. There are a small number of animal studies that have examined the effects of surgery on cognitive function. Wan et al showed that orthopedic surgery, in wild-type mice under an intravenous anesthesia, induced postoperative cognitive impairment, while the anesthetic alone did not. ${ }^{61}$ Similarly, Tang et al compared desflurane alone versus desflurane with cecal ligation in transgenic mice and found that surgery per se led to memory impairment, enhanced tau pathology, and neuroinflammation. ${ }^{62}$ Interestingly, this memory deficit can be detected out until at least 3 months following the surgical procedure. In another study, major surgery (partial hepatectomy) was found to provoke gliosis, $\beta$-amyloid accumulation, tau phosphorylation, and cognitive impairment in older mice, while sham surgery did not. ${ }^{63}$ Taken together, these results demonstrate that cognitive deficits may largely be a result of surgery rather than due to specific effects of anesthesia. However, the exact mechanism that mediates surgery-induced cognitive impairment remains unclear. It is hypothesized that neuroinflammation induced by surgery is closely associated with cognitive impairment. ${ }^{64}$ For example, in animal studies, surgery has been found to activate glial cells and to induce an excessive release of TNF- $\alpha$ and IL-1 $\beta$ in the brain which underlie the cognitive deficits. ${ }^{65,66}$ Interestingly, anti-inflammatory drugs have been found to prevent surgery-induced cognitive dysfunction and changes in $\beta$-amyloid and tau processing. ${ }^{63}$ Therefore, the results of the preclinical studies that have been published suggest that both anesthetic agents and surgery can independently lead to cognitive impairment. Nonetheless, the few animal studies have provided evidence that the effect due to surgery per se appears to be dominant.

\section{Clinical studies Biomarker studies}

Human data are essential for determining whether anesthesia and surgery induce cognitive deficits, although no definitive evidence has been obtained to date. In particular, the long period of time over which AD pathogenesis develops in the absence of cognitive symptoms has made human studies difficult to conduct. It is also difficult to examine the impact of anesthesia on the development of AD pathology in human studies. Thus, human biomarker research is a valuable approach for examining the interactions between anesthesia, surgery, and AD neuropathology.

A small number of studies have examined changes in the levels of cerebrospinal fluid (CSF) biomarkers during the postoperative period. In one study, Palotás et al showed that level of CSF A $\beta$ was significantly decreased, whereas the levels of injury biomarkers (S100B and tau) were elevated at 6 months in patients who underwent coronary artery bypass surgery (CABG). ${ }^{67}$ These findings demonstrated postsurgical cognitive impairment associated with changes in CSF biomarkers similar to that found in AD. In another study, Tang et al found that the total-tau/A $\beta_{1-42}$ ratio in CSF increased postoperatively in patients who underwent idiopathic nasal 
CSF leak repair, and these changes were consistent with those observed in AD. ${ }^{68}$ Furthermore, in the latter study, elevated levels of the proinflammatory cytokines, IL-6, TNF- $\alpha$, and IL-10, were also detected in the CSF, indicating that a neuroinflammatory response was elicited by the anesthesia and surgery. Nonetheless, the limitations of these studies include small sample sizes, an absence of control groups, and short follow-up periods. Although the postoperative CSF biomarkers change in a pattern consistent with $\mathrm{AD}$, further studies are warranted to better understand the evolution of biomarkers and their relevance to the long-term consequences of AD pathology.

\section{Retrospective case-control studies}

In order to examine the relationship between prior exposure to general anesthesia (GA) and subsequent risk of dementia, evidence from observational human studies is important. Many case-control studies have been conducted, and they have come to disparate conclusions whether GA is a potential risk factor for dementia. It is possible that small cohorts, biased study populations, and confounding coincident illnesses have contributed to these contradictory results. A meta-analysis of 15 case-control studies included a total of 1,752 cases and 5,261 controls, and the inclusion criteria for the case control studies included the use of standardized clinical criteria for $\mathrm{AD}$ or dementia. ${ }^{69,70}$ In addition, the control groups included either patients who underwent surgery with regional anesthesia or those who had no history of surgery. However, there was no statistically significant association between GA and the development of $\mathrm{AD}$ (pooled $\mathrm{OR}=1.05,95 \%$ confidence interval $[\mathrm{CI}]=0.93-1.19 ; P=0.43)$. In a retrospective population-based nested case-control study conducted by Sprung et al that used data from the Rochester Epidemiology Project and the Mayo Clinic Alzheimer's Disease Patient Registry, case groups included 877 patients with incident dementia recorded between 1985 and 1994, while the control groups included sex- and age-matched individuals who did not have a diagnosis of dementia. A total of $70 \%$ of the dementia patients had been exposed to GA compared with $72.5 \%$ of the control group, and there was no significant association between exposure to GA after age 45 years and the diagnosis of dementia $(\mathrm{OR}=0.89,95 \% \mathrm{CI}=0.73-1.10$, $P=0.27) .{ }^{15}$ Similarly, Chen et al published a populationbased case-control study using the Taiwan Longitudinal Health Insurance Database, which contains claims data for one million residents who are covered by Taiwan's universal health insurance. ${ }^{16}$ This study included 5,345 patients who were newly diagnosed with dementia and who were older than 50 years and 21,380 individuals without dementia from 2005 to 2009. The two groups were matched for age, sex, and index date. In addition, GA exposure was categorized into three subtypes: endotracheal tube intubation GA (ETGA), intravenous injection GA (IVGA), or intramuscular injection GA (IMGA), versus heavy sedation. The dementia group had higher rates of exposure to ETGA and IVGA/IMGA compared with the control group, whereas the rates of exposure to heavy sedation did not differ between the groups. For the individuals exposed to surgery under ETGA (OR $=1.34$, 95\% CI $=1.25-1.44)$ or IVGA/IMGA (OR $=1.28,95 \% \mathrm{CI}$ $=1.14-1.43$ ), they were found to be at significantly higher risk of developing dementia and a dose-dependent response was observed $(P<0.0001)$.

\section{Cohort studies}

Lee et al performed a retrospective cohort analysis to examine if $\mathrm{CABG}$ and $\mathrm{GA}$ are associated with an earlier emergence of AD. ${ }^{12}$ The study population included individuals older than 55 years without a diagnosis of dementia prior to surgery. One group underwent GA and $\mathrm{CABG}$, while the other group received only sedation, for percutaneous transluminal coronary angioplasty. During the 5-year follow-up period, patients who had undergone CABG and GA had a 1.7-fold increased risk of developing $\mathrm{AD}$ compared with those who only received sedation and percutaneous transluminal coronary angioplasty $(P=0.04)$. In a subsequent population-based retrospective cohort study, data from the Taiwan Longitudinal Health Insurance Database was used to obtain a cohort that included 24,901 patients $\geq 50$ years who, since 1995 , were anesthetized for the first time from 2004 to $2007 .{ }^{71}$ The ageand sex-matched control group consisted of 110,972 patients who had not been exposed to anesthesia. The first occurrence of a dementia diagnosis was recorded, and to minimize the possibility of misdiagnosing POCD as dementia, the diagnosis had to be recorded twice, with the first occurrence being at least 3 months after the administration of anesthesia. After a follow-up period of 3-7 years, the risk of dementia in the anesthesia group was found to be significantly higher than that in the control group (hazard ratio $[\mathrm{HR}]=1.99,95 \% \mathrm{CI}$ $=1.81-2.17 ; P<0.001)$. Furthermore, both exposure to GA and to regional anesthesia yielded increased risks of incident dementia $(\mathrm{HR}=1.46[95 \% \mathrm{CI}=1.28-1.68]$ and $\mathrm{HR}=1.80$ [95\% CI $=1.28-1.68]$, respectively; $P<0.001)$. The results of this study suggest that patients who undergo anesthesia and surgery may be at increased risk for developing dementia. However, given the population-based analysis design of the study, the large sample size, and the long follow-up period 
that provided adequate power for this study, a causal relationship could not be determined by the positive association alone.

\section{Prospective studies}

To investigate the associations between anesthesia, surgery, and $\mathrm{AD}$, prospective randomized controlled trials are not always indicated nor are ethical to conduct. Thus, there are very few prospective human studies that have investigated the association between anesthesia and AD. Liu et al published a prospective randomized, parallel-group study to evaluate whether exposure to anesthetics induces progression of amnestic mild cognitive impairment (aMCI) in a Chinese population. ${ }^{72}$ The study group consisted of 180 aMCI patients who were randomly assigned to receive sevoflurane, propofol, or lidocaine epidural anesthesia for lumbar spinal surgery. Sixty aMCI outpatients served as the control group. At the 2-year follow-up, the number of aMCI patients who progressed to $\mathrm{AD}$ did not differ between the groups. However, the number of patients who exhibited progression of aMCI (which was assessed based on changes detected in neuropsychological tests that were performed) was greater in the sevoflurane group than in the control group $(P<0.005)$, yet was not greater in the propofol or lidocaine groups. The authors concluded that sevoflurane anesthesia for lumbar spine surgery may accelerate cognitive decline, although the relatively small sample size and short follow-up period were acknowledged as limitations of the study.

\section{Conclusion}

The results of both in vitro and animal studies suggest that GA, especially inhaled anesthesia, and surgery can accelerate $\mathrm{A} \beta$ production, tau hyperphosphorylation, and $\mathrm{AD}$ pathology. Cognitive changes, including postoperative delirium and POCD, have been well-defined in the elderly, although these were not discussed in detail here. Nonetheless, it remains unclear whether anesthesia and surgery can increase the risk of long-term cognitive impairment conditions such as dementia and AD. Moreover, in clinical settings and human research, anesthesia and surgery remain inseparable. It is also difficult and unethical to conduct prospective, large sample-sized, randomized controlled trials to evaluate the association between anesthesia and/or surgery and AD. To date, the results of observational studies conducted in humans regarding potential associations between anesthesia, surgery, and AD have been inconsistent. A previously published metaanalysis showed no increased risk of AD following exposure to GA and surgery in case-controlled studies. ${ }^{69}$ However, studies that have been published more recently do suggest a possible association between anesthesia and surgery and the development of dementia. ${ }^{15,71}$ Moreover, the results of a prospective randomized controlled trial suggest that sevoflurane may accelerate cognitive decline in patients with aMCI, although the relatively small sample size and short follow-up period limit the strengths of these results, and further studies are warranted. ${ }^{72}$

Given the gaps identified in the current preclinical and clinical research investigations that have been published, further studies are needed to confirm the clinical relevance of the in vitro findings regarding the relationship between anesthesia and/or surgery and neurodegenerative complications. In addition, well designed and adequately powered prospective and retrospective human studies with longer follow-up periods are needed to elucidate whether exposure to anesthesia and/or surgery are causally associated with the development of dementia.

\section{Disclosure}

The authors report no conflicts of interest in this work.

\section{References}

1. Ferri CP, Prince M, Brayne C, Alzheimer's Disease International, et al. Global prevalence of dementia: a Delphi consensus study. Lancet. 2006;366:2112-2117.

2. Zimmerman S, Sloane PD, Williams CS, et al. Dementia care and quality of life in assisted living and nursing homes. Gerontologist. 2005;45(1):133-146.

3. Canadian study of health and aging: study methods and prevalence of dementia. CMAJ. 1994;150:899-913.

4. Querfurth HW, LaFerla FM. Alzheimer's disease. $N$ Engl $J$ Med. 2010;362:329-344.

5. Eckenhoff RG, Johansson JS, Wei H, et al. Inhaled anesthetic enhancement of amyloid-beta oligomerization and cytotoxicity. Anesthesiology. 2004;101:703-709.

6. Mandal PK, Fodale V. Isoflurane and desflurane at clinically relevant concentrations induce amyloid betapeptide oligomerization: an NMR study. Biochem Biophys Res Commun. 2009;379:716-720.

7. Culley DJ, Baxter MG, Crosby CA, Yukhananov R, Crosby G. Longterm impairment of acquisition of a spatial memory task following isoflurane-nitrous oxide anesthesia in rats. Anesthesiology. 2004;100: 309-314.

8. Planel E, Richter KEG, Nolan CE, et al. Anesthesia leads to tau hyperphosphorylation through inhibition of phosphatase activity by hypothermia. J Neurosci. 2007;27:3090-3097.

9. Xie Z, Culley DJ, Dong Y, et al. The common inhalation anesthetic isoflurane induces caspase activation and increases amyloid beta-protein level in vivo. Ann Neurol. 2008;64:618-627.

10. Deiner S, Silverstein JH. Postoperative delirium and cognitive dysfunction. Br J Anaesth. 2009;103(Suppl 1):i41-i46.

11. Nadelson MR, Sanders RD, Avidan MS. Perioperative cognitive trajectory in adults. Br J Anaesth. 2014;112(3):440-451.

12. Lee TA, Wolozin B, Weiss KB, Bednar MM. Assessment of the emergence of Alzheimer's disease following coronary artery bypass graft surgery or percutaneous transluminal coronary angioplasty. J Alzheimers Dis. 2005;7:319-324. 
13. Knopman DS, Petersen RC, Cha RH, Edland SD, Rocca WA. Coronary artery bypass grafting is not a risk factor for dementia or Alzheimer disease. Neurology. 2005;65:986-990.

14. Selnes OA, Royall RM, Grega MA, Borowicz LM Jr, Quaskey S, McKhann GM. Cognitive changes 5 years after coronary artery bypass grafting: is there evidence of late decline? Arch Neurol. 2001; 58(4):598-604.

15. Sprung J, Jankowski CJ, Roberts RO, et al. Anesthesia and incident dementia: a population-based, nested, case-control study. Mayo Clin Proc. 2013;88:552-561.

16. Chen CW, Lin CC, Chen KB, Kuo YC, Li CY, Chung CJ. Increased risk of dementia in people with previous exposure to general anesthesia: a nationwide population-based case-control study. Alzheimers Dement. 2014;10:196-204.

17. Baranov D, Bickler PE, Crosby GJ, et al; First International Workshop on Anesthetics and Alzheimer's Disease. Consensus statement: first international workshop on anesthetics and Alzheimer's disease. Anesth Analg. 2009;108:1627-1630.

18. Finder VH. Alzheimer's disease: a general introduction and pathomechanism. J Alzheimers Dis. 2010;22(Suppl 3):5-19.

19. Yan R, Vassar R. Targeting the $\beta$ secretase BACE1 for Alzheimer's disease therapy. Lancet Neurol. 2014;13(3):319-329.

20. Kumar A, Dogra S. Neuropathology and therapeutic management of Alzheimer's disease - an update. Drugs Future. 2008;33(5):433-446.

21. Kumar A, Singh A, Ekavali. A review on Alzheimer's disease pathophysiology and its management: an update. Pharmacol Rep. 2015; 67(2):195-203.

22. Hardy J, Selkoe DJ. The amyloid hypothesis of Alzheimer's disease: progress and problems on the road to therapeutics. Science. 2002;297: 353-356.

23. Wu X, Lu Y, Dong Y, et al. The inhalation anesthetic isoflurane increases levels of proinflammatory TNF-alpha, IL-6, and IL-1beta. Neurobiol Aging. 2012;33:1364-1378.

24. Mandal PK, Pettegrew JW. Abeta peptide interactions with isoflurane, propofol, thiopental and combined thiopental with halothane: a NMR study. Biochim Biophys Acta. 2008;1778:2633-2639.

25. Mena MÁ, Perucho J, Rubio I, de Yébenes JG. Studies in animal models of the effects of anesthetics on behavior, biochemistry, and neuronal cell death. J Alzheimers Dis. 2010;22(Suppl 3):43-48.

26. Xie Z, Dong Y, Maeda U, et al. The common inhalation anesthetic isoflurane induces apoptosis and increases amyloid beta protein levels. Anesthesiology. 2006;104:988-994.

27. Dong Y, Zhang G, Zhang B, et al. The common inhalational anesthetic sevoflurane induces apoptosis and increases beta-amyloid protein levels. Arch Neurol. 2009;66:620-631.

28. Wei H, Xie Z. Anesthesia, calcium homeostasis and Alzheimer's disease. Curr Alzheimer Res. 2009;6:30-35.

29. Xie Z, Dong Y, Maeda U, et al. The inhalation anesthetic isoflurane induces a vicious cycle of apoptosis and amyloid beta-protein accumulation. J Neurosci. 2007;27:1247-1254.

30. Dong Y, Xu Z, Zhang Y, et al. RNA interference-mediated silencing of BACE and APP attenuates the isoflurane-induced caspase activation. Med Gas Res. 2011;1:5.

31. Bianchi SL, Tran T, Liu C, et al. Brain and behavior changes in 12-month-old Tg2576 and nontransgenic mice exposed to anesthetics. Neurobiol Aging. 2008;29:1002-1010.

32. Perucho J, Rubio I, Casarejos MJ, et al. Anesthesia with isoflurane increases amyloid pathology in mice models of Alzheimer's disease. J Alzheimers Dis. 2010;19:1245-1257.

33. Zhang B, Dong Y, Zhang G, et al. The inhalation anesthetic desflurane induces caspase activation and increases amyloid beta-protein levels under hypoxic conditions. J Biol Chem. 2008;283:11866-11875.

34. Zhang Y, Dong Y, Wu X, et al. The mitochondrial pathway of anesthetic isoflurane-induced apoptosis. J Biol Chem. 2010;285:4025-4037.

35. Zhen Y, Dong Y, Wu X, et al. Nitrous oxide plus isoflurane induces apoptosis and increases beta-amyloid protein levels. Anesthesiology. 2009; 111:741-752.
36. Zhang Y, Xu Z, Wang $\mathrm{H}$, et al. Anesthetics isoflurane and desflurane differently affect mitochondrial function, learning, and memory. Ann Neurol. 2012;71:687-698.

37. Xie Z, Xu Z. General anesthetics and $\beta$-amyloid protein. Prog Neuropsychopharmacol Biol Psychiatry. 2013;47:140-146.

38. Buee L, Bussiere T, Buee-Scherrer V, Delacourte A, Hof PR. Tau protein isoforms, phosphorylation and role in neurodegenerative disorders. Brain Res Brain Res Rev. 2000;33:95-130.

39. Bloom GS. Amyloid- $\beta$ and tau: the trigger and bullet in Alzheimer disease pathogenesis. JAMA Neurol. 2014;71(4):505-508.

40. Tan W, Cao X, Wang J, Lv H, Wu B, Ma H. Tau hyperphosphorylation is associated with memory impairment after exposure to $1.5 \%$ isoflurane without temperature maintenance in rats. Eur J Anaesthesiol. 2010;27: 835-841.

41. Lewis J, McGowan E, Rockwood J, et al. Neurofibrillary tangles, amyotrophy and progressive motor disturbance in mice expressing mutant (P301L) tau protein. Nat Genet. 2000;25:402-405.

42. Menuet $\mathrm{C}$, Borghgraef $\mathrm{P}$, Voituron N, et al. Isoflurane anesthesia precipitates tauopathy and upper airways dysfunction in pre-symptomatic Tau.P301L mice: possible implication for neurodegenerative diseases. Neurobiol Dis. 2012;46:234-243.

43. Run X, Liang Z, Zhang L, Iqbal K, Grundke-Iqbal I, Gong CX. Anesthesia induces phosphorylation of tau. JAlzheimers Dis. 2009;16: 619-626.

44. Whittington RA, Bretteville A, Dickler MF, Planel E. Anesthesia and tau pathology. Prog Neuropsychopharmacol Biol Psychiatry. 2013;47: $147-155$.

45. Whittington RA, Virág L, Marcouiller F, et al. Propofol directly increases tau phosphorylation. PLoS One. 2011;6:e16648.

46. Le Freche H, Brouillette J, Fernandez-Gomez FJ, et al. Tau phosphorylation and sevoflurane anesthesia: an association to postoperative cognitive impairment. Anesthesiology. 2012;116:779-787.

47. Tang JX, Mardini F, Caltagarone BM, et al. Anesthesia in presymptomatic Alzheimer's disease: a study using the triple-transgenic mouse model. Alzheimers Dement. 2011;7:521-531.

48. Heneka MT, Carson MJ, El Khoury J, et al. Neuroinflammation in Alzheimer's disease. Lancet Neurol. 2015;14(4):388-405.

49. Quintanilla RA, Orellana DI, Gonzalez-Billault C, Maccioni RB. Interleukin-6 induces Alzheimer-type phosphorylation of tau protein by deregulating the cdk5/p35 pathway. Exp Cell Res. 2004;295: 245-257.

50. Perry VH, Cunningham C, Holmes C. Systemic infections and inflammation affect chronic neurodegeneration. Nat Rev Immunol. 2007;7: 161-167.

51. Lucin KM, Wyss-Coray T. Immune activation in brain aging and neurodegeneration: too much or too little? Neuron. 2009;64:110-122.

52. Kitazawa M, Oddo S, Yamasaki TR, Green KN, LaFerla FM. Lipopolysaccharide-induced inflammation exacerbates tau pathology by a cyclin-dependent kinase 5-mediated pathway in a transgenic model of Alzheimer's disease. J Neurosci. 2005;25:8843-8853.

53. Acharya NK, Goldwaser EL, Forsberg MM, et al. Sevoflurane and isoflurane induce structural changes in brain vascular endothelial cells and increase blood-brain barrier permeability: possible link to postoperative delirium and cognitive decline. Brain Res. 2015;1620:29-41.

54. Lehmberg J, Waldner M, Baethmann A, Uhl E. Inflammatory response to nitrous oxide in the central nervous system. Brain Res. 2008; 1246:88-95.

55. Zhang H, Astrof NS, Liu JH, Wang JH, Shimaoka M. Crystal structure of isoflurane bound to integrin LFA-1 supports a unified mechanism of volatile anesthetic action in the immune and central nervous systems. FASEB J. 2009;23(8):2735-2740.

56. Yuki K, Astrof NS, Bracken C, Soriano SG, Shimaoka M. Sevoflurane binds and allosterically blocks integrin lymphocyte function-associated antigen-1. Anesthesiology. 2010;113(3):600-609.

57. Tang JX, Eckenhoff MF, Eckenhoff RG. Anesthetic modulation of neuroinflammation in Alzheimer's disease. Curr Opin Anaesthesiol. 2011;24(4):389-394. 
58. Luo X, Yang L, Chen X, Li S. Tau hyperphosphorylation: a downstream effector of isoflurane-induced neuroinflammation in aged rodents. Med Hypotheses. 2014;82(1):94-96.

59. Cao L, Li L, Lin D, Zuo Z. Isoflurane induces learning impairment that is mediated by interleukin 1beta in rodents. PLoS One. 2012;7:e51431.

60. Li SY, Xia LX, Zhao YL, et al. Minocycline mitigates isoflurane-induced cognitive impairment in aged rats. Brain Res. 2013;1496:84-93.

61. Wan Y, Xu J, Ma D, Zeng Y, Cibelli M, Maze M. Postoperative impairment of cognitive function in rats: a possible role for cytokinemediated inflammation in the hippocampus. Anesthesiology. 2007;106: 436-443.

62. Tang JX, Mardini F, Janik LS, et al. Modulation of murine Alzheimer pathogenesis and behavior by surgery. Ann Surg. 2013;257:439-448.

63. Wan Y, Xu J, Meng F, et al. Cognitive decline following major surgery is associated with gliosis, $\beta$-amyloid accumulation, and tau phosphorylation in old mice. Crit Care Med. 2010;38(11):2190-2198.

64. Eckenhoff RG, Laudansky KF. Anesthesia, surgery, illness and Alzheimer's disease. Prog Neuropsychopharmacol Biol Psychiatry. 2013;47:162-166.

65. Cibelli M, Fidalgo AR, Terrando N, et al. Role of interleukin-1beta in postoperative cognitive dysfunction. Ann Neurol. 2010;68:360-368.
66. Terrando N, Monaco C, Ma D, Foxwell BM, Feldmann M, Maze M. Tumor necrosis factor-alpha triggers a cytokine cascade yielding postoperative cognitive decline. Proc Natl Acad Sci U S A. 2010;107: 20518-22022.

67. Palotás A, Reis HJ, Bogáts G, et al. Coronary artery bypass surgery provokes Alzheimer's disease-like changes in the cerebrospinal fluid. J Alzheimers Dis. 2010;21:1153-1164.

68. Tang JX, Baranov D, Hammond M, Shaw LM, Eckenhoff MF, Eckenhoff RG. Human Alzheimer and inflammation biomarkers after anesthesia and surgery. Anesthesiology. 2011;115:727-732.

69. Seitz DP, Shah PS, Herrmann N, Beyene J, Siddiqui N. Exposure to general anesthesia and risk of Alzheimer's disease: a systematic review and meta-analysis. BMC Geriatr. 2011;11:83-90.

70. Hussain M, Berger M, Eckenhoff RG, Seitz DP. General anesthetic and the risk of dementia in elderly patients: current insights. Clin Interv Aging. 2014;9:1619-1628.

71. Chen PL, Yang CW, Tseng YK, et al. Risk of dementia after anaesthesia and surgery. Br J Psychiatry. 2014;204:188-193.

72. Liu Y, Pan N, Ma Y, et al. Inhaled sevoflurane may promote progression of amnestic mild cognitive impairment: a prospective, randomized parallel-group study. Am J Med Sci. 2013;345(5):355-360.
Journal of Pain Research

\section{Publish your work in this journal}

The Journal of Pain Research is an international, peer-reviewed, open access, online journal that welcomes laboratory and clinical findings in the fields of pain research and the prevention and management of pain. Original research, reviews, symposium reports, hypothesis formation and commentaries are all considered for publication.

\section{Dovepress}

The manuscript management system is completely online and includes a very quick and fair peer-review system, which is all easy to use. Visit http://www.dovepress.com/testimonials.php to read real quotes from published authors. 